\title{
INFRAESTRUCTURA Y ACCESO A TECNOLOGÍAS DIGITALES COMO CONDICIONANTES DE LAS PRÁCTICAS PEDAGÓGICAS DE LOS PROFESORES DE SANTA BÁRBARA, HONDURAS
}

\author{
Cinthia MARgarita SABillón JimÉnEZ² \\ Universidade Federal da Bahia, Brasil / sabillon86@gmail.com \\ María Helena BONiLla \\ Universidade Federal da Bahia, Brasil / bonillabr@gmail.com
}

Recibido: 1/4/2019/ Aceptado: 14/6/2019

doi: 10.26439/contratexto2019.n032.4621

Resumen. Las sociedades contemporáneas se caracterizan por experimentar, a una gran velocidad e intensidad, constantes cambios tecnológicos y sociales. Sin embargo, los ambientes escolares no siempre logran acompañar e incorporar debidamente esos cambios, lo que puede verse reflejado en sus prácticas pedagógicas. Dicho desfase en el acompañamiento e incorporación puede deberse a diversos factores, sin embargo, a pesar de ello, en Honduras existen algunas experiencias de prácticas pedagógicas con tecnologías digitales por parte de profesores, con el objeto de acompañar dichos cambios tecnológicos y sociales. El presente artículo analiza y discute los resultados de una investigación de maestría en educación, en la que se estudiaron las prácticas pedagógicas con tecnologías digitales. La discusión está centrada en uno de sus factores condicionantes, con el objetivo de comprender de qué manera la infraestructura y el acceso a tecnologías digitales condicionan el desarrollo de prácticas pedagógicas de los profesores de Santa Bárbara, Honduras. La investigación fue de naturaleza cualitativa, con método de estudio de caso, y los datos fueron sometidos al análisis textual discursivo. Los resultados indican que la infraestructura de los colegios junto con la

\footnotetext{
1 El presente trabajo fue realizado con apoyo de la Coordenação de Aperfeiçoamento de Pessoal de Nível Superior Brasil (Capes) - Código de Financiamiento 001

2 Doctoranda en educación por la Universidade Federal da Bahia, Brasil. https://orcid.org/0000-0003-4270-1705 3 Doctora en Educación por la Universidade Federal da Bahia, Brasil. https://orcid.org/0000-0002-0137-6363
} 
exclusión digital dificultan la implementación de las tecnologías digitales en las prácticas pedagógicas.

Palabras clave: tecnologías digitales / profesor / prácticas pedagógicas / infraestructura / inclusión digital

\title{
INFRASTRUCTURE AND ACCESS TO DIGITAL TECHNOLOGIES AS DETERMINANTS OF THE PEDAGOGICAL PRACTICES OF TEACHERS FROM SANTA BÁRBARA, HONDURAS
}

\begin{abstract}
Contemporary societies typically experience constant technological and social changes at a great speed and intensity. However, school environments do not always manage to adequately support and incorporate those changes, which may be reflected in their pedagogical practices. This gap in supporting and incorporating said changes may be due to various factors; however, despite of them, there have been some experiences of pedagogical practices with digital technologies carried out by teachers in Honduras to support those technological and social changes. This article analyzes and discusses the results of a master's research in education, where pedagogical practices with digital technologies were studied. The discussion is centered in one of its determining factors in order to understand how infrastructure and access to digital technologies condition the development of pedagogical practices by teachers from Santa Bárbara, Honduras. The research was qualitative in nature and used the case study method. Data analysis was made using the textual-discursive method. The results indicated that schools' infrastructure, together with digital exclusion, make it difficult to implement digital technologies in pedagogical practices.
\end{abstract}

Keywords: digital technologies / teacher / pedagogical practices / infrastructure / digital inclusion 


\section{INFRAESTRUTURA E ACESSO ÀS TECNOLOGIAS DIGITAIS COMO CONDICIONANTE DAS PRÁTICAS PEDAGÓGICAS DOS PROFESSORES DE SANTA BÁRBARA, HONDURAS}

Resumo. As sociedades contemporâneas caracterizam-se por experimentar constantes mudanças tecnológicas e sociais em grande velocidade e intensidade. No entanto, nem sempre os ambientes escolares conseguem acompanhar e incorporar adequadamente essas mudanças, o que pode ser refletido em suas práticas pedagógicas. Esta lacuna no acompanhamento e incorporação pode dever-se a vários fatores, contudo, em Honduras existem algumas experiências de práticas de ensino com tecnologias digitais por parte dos professores que pretendem acompanhar essas mudanças tecnológicas e sociais. Este artigo analisa e discute os resultados de uma pesquisa de mestrado em educação, em que foram estudadas práticas pedagógicas com tecnologias digitais. A discussão está centrada em um dos seus condicionantes com o objetivo de compreender a maneira em que a infraestrutura e o acesso às tecnologias digitais influenciam o desenvolvimento de práticas pedagógicas de professores em Santa Bárbara, Honduras. A pesquisa foi de natureza qualitativa, com o método de estudo de caso e os dados foram analisados por meio de análise discursiva textual. Os resultados indicam que a infraestrutura das escolas aliada à exclusão digital, dificulta a implementação de tecnologias digitais com práticas pedagógicas.

Palavras chave: tecnologias digitais / professor / práticas pedagógicas / infraestrutura / inclusão digital 


\section{INTRODUCCIÓN}

Las tecnologías digitales (TD) van adquiriendo un papel preponderante en el cotidiano de los individuos, generando cambios a grandes velocidades e intensidades, y resulta difícil no tener algún tipo de vínculo con ellas; de manera directa o indirecta se establecen ciertos tipos de nexos entre los individuos y las TD desde el contacto directo con un computador o con las informaciones transmitidas por un satélite, hasta el contacto que puede tener cualquier individuo con una publicidad que se encuentre en la calle (materiales publicitarios creados en un computador). Sin embargo, esa velocidad e intensidad no se ve reflejada de la misma manera en los ambientes educativos.

Es fundamental que la escuela logre acompañar los cambios que la sociedad experimenta producto de la presencia de las TD. Para eso es preciso que la práctica pedagógica vaya acompañada en todo momento de un proceso de reflexión colectiva constante, que permita generar las acciones educativas que garanticen que los individuos puedan desempeñarse dentro de la sociedad contemporánea. "En ese aspecto, una práctica pedagógica, en su sentido de praxis, se configura como una acción consciente y participativa, que emerge de la multidimensionalidad que cerca el acto educativo" (Franco, 2016, p. 536).

Para Barragán (2012), la práctica pedagógica no se reduce a una simple replicabilidad de técnicas aprendidas en los diversos procesos formativos, sino que es una cuestión más de disposición humana, principalmente por esa multidimensionalidad que impera en los ambientes educativos. Es esa disposición humana que permitirá en cierta medida que el profesor pueda ir acompañando los cambios generados por las TD en la sociedad, para luego readecuar su práctica pedagógica a esa realidad. Es claro que esa responsabilidad no es exclusiva del profesor, también atañe a las autoridades educativas, las cuales deben tener esas disponibilidades para que puedan contribuir a generar un mejor panorama para la implementación de las TD en los ambientes educativos.

El proceso de acompañamiento de las prácticas pedagógicas, a los cambios generados por las TD, puede conducir a una simple sustitución de los recursos didácticos análogos por los digitales, haciendo que la implementación de las TD sea desde una perspectiva tecnicista, considerándolas únicamente como complementos de la acción docente. Por otro lado, si se llega a ver a las TD como nuevos escenarios en los que podemos ejercer la acción docente, orientada principalmente hacia la producción/ distribución de conocimiento, tanto por parte de alumnos como de profesores, se tendrá un mayor aprovechamiento de las potencialidades de las TD.

Esa transformación en las prácticas pedagógicas es importante debido a que ellas constituyen al mismo tiempo prácticas sociales que conducen hacia una práctica 
educativa, lo que termina repercutiendo en la sociedad. Las prácticas pedagógicas se organizan intencionalmente para alcanzar las expectativas educacionales, que pueden ser implantadas, solicitadas o requeridas por la sociedad en la que se encuentran inseridos los individuos (Franco, 2016). Para Franco (2016), las configuraciones que se dan en las prácticas pedagógicas generan las prácticas educativas, las cuales no pueden estar aisladas ni desligadas de todo el conjunto pedagógico, estas deben estar fundamentadas en las prácticas pedagógicas, que les adjudican sentido y dirección.

Sin embargo, las prácticas pedagógicas se ven condicionadas por una serie de factores, políticas públicas/institucionales, la formación de los profesores, percepción sobre las TD, infraestructura, acceso a TD, entre otros. Cada uno de estos aspectos influye de diversas maneras en el desempeño de los profesores, condicionando las prácticas pedagógicas con TD. En el caso específico de la infraestructura de los centros educativos, incluyendo las condiciones físicas de los predios, como también las mismas TD, representa un aspecto muy importante al momento de pensar y planificar la labor docente.

La llegada de las TD a los ambientes educativos representó una serie de problemáticas para las escuelas, principalmente al no contar con las condiciones adecuadas para la recepción de los diversos dispositivos digitales lanzados en el mercado y que son introducidos a los centros educativos por diversos medios, ya sea por los alumnos, profesores o inclusive políticas públicas o institucionales. Pero, a pesar de las condiciones de infraestructura de las escuelas, las TD están inseridas en el cotidiano escolar, y tienen diversas recepciones/percepciones por parte de profesores y autoridades educativas.

Algunos análisis basados en los resultados del Segundo Estudio Regional Comparativo y Explicativo (Serce) (Organización de las Naciones Unidas para la Educación, la Ciencia y la Cultura, Unesco, 2006), sugieren que si las escuelas contaran con mejores instalaciones y servicios básicos, podrían condicionar un ambiente propicio para lograr mejores resultados de aprendizaje. En base a la importancia que tiene la infraestructura en los procesos educativos, la inversión de tiempo y dinero en estos aspectos no constituye un lujo, sino una necesidad (Duarte, Gargiulo y Moreno, 2011), por lo que pensar en la modernización de la infraestructura de los centros educativos es una problemática latente, que necesita ser solucionada, para que pueda dar cuenta de las exigencias generadas por la presencia de las TD en las escuelas.

La infraestructura escolar está constituida por todos los recursos materiales que las instituciones educativas disponen. En este aspecto, abarca desde el diseño de las aulas de clases, como de los escritorios, sillas, pupitres, librerías, computadores, instalaciones eléctricas, de internet, etc. Todos estos elementos pueden contribuir o dificultar en ocasiones las prácticas pedagógicas. Pero no solo basta el tener los 
recursos materiales, sino que hay que discutir lo referente a la calidad de los mismos, no basta el tener un espacio, sino ver las condiciones en las que se encuentran, o tener una amplia biblioteca sin pensar que dispone de libros actuales de diversas temáticas, o tener conexión a internet sin discutir el ancho de banda o las restricciones de acceso que pueden tener en ocasiones.

Para tener un verdadero proceso de inclusión digital ${ }^{4}$, no basta el simple acceso, sino que es necesario tener una calidad de ancho de banda que permita el poder tener acceso y disponer todo tipo de información en la red. Para Sérgio Amadeu da Silveira (2001), la exclusión digital está dificultando que la exclusión social se disminuya, ya que muchas de las actividades que los ciudadanos realizan están mediadas por TD, por ejemplo, actividades económicas, gubernamentales, y hasta mucha de la producción cultural de los grupos sociales están mediadas por las TD. La brecha digital existente en las sociedades contemporáneas está ligada directamente a la exclusión social, a los sectores sociales menos favorecidos económicamente se les dificulta el tener acceso a TD y, por ende, a las informaciones disponibles en internet.

Esta realidad latente en Honduras se ve reflejada en la Visión de País, en donde se establece la intención de equipar con tecnologías y conectividad para enfrentar la baja calidad del entorno escolar, debido a que "[...] es manifiesta la desigualdad en el acceso a información y tecnologías de parte del educando que asiste a escuelas desprovistas de materiales y equipos, que sí existen de manera generalizada en escuelas y colegios del subsector privado" (República de Honduras, 2010, p. 67).

Ante la importancia que tienen las TD en la sociedad contemporánea y con la intención de disminuir la brecha digital en Honduras, se han ido implementando algunas medidas para formar a los hondureños con las habilidades que les permitan desempeñarse en el actual contexto. Uno de los programas creados por el Gobierno de Honduras es El Internet del Pueblo, que se enmarca en el primer y tercer eje de la Agenda Digital de Honduras. El proyecto El Internet del Pueblo consiste en brindar conexión de internet a los hondureños en escuelas y parques o plazas de Honduras, con el propósito de disminuir la brecha digital existente en el territorio; para el 2016 se habían logrado instalar 2500 puntos de acceso de internet gratuito en centros educativos públicos, en 143 parques o plazas públicas y en 34 centros de enseñanza no formales. Sin embargo, el acceso a esta red tiene restricciones, impidiendo que las personas puedan ingresar a determinados sitios web, además el ancho de banda que ofrece no es suficiente para los centros educativos; las velocidades de El Internet del Pueblo son: fibra óptica de tres

4 La inclusión digital es más un discurso, que un concepto, el cual se ha venido utilizando por organizaciones internacionales y se ha insertado en los discursos de los gobiernos y programas de cada país (Bonilla y Pretto, 2011). 
a cinco Mbps, satelital $128-512$ Kbps, WIMAX 3 Mbps, y radio con enlace de tres a cinco Mbps (Dirección de Tecnologías de la Información y Comunicación-Ditic, comunicación por e-mail, 6 de julio del 2016)5.

Por su parte la Secretaría de Educación de Honduras también ha realizado proyectos encaminados a la incorporación de las TD en el ámbito educativo. Un ejemplo de eso lo representa el Sistema de Administración de Centros (SACE, s. f.), implementado desde el 2013, el cual consiste en una plataforma donde se administran informaciones de todos los centros educativos del país, datos como matrículas de alumnos, calificaciones, alumnos desertores, horarios de clases, perfiles de los profesores, entre otros aspectos. Todos esos datos están digitalizados en la plataforma del SACE, cada profesor tiene un usuario y contraseña para poder ingresar las calificaciones y editar su perfil profesional, lo mismo sucede con los directores de las escuelas, quienes dejan disponible en la plataforma el resto de las informaciones de cada centro educativo.

Otro elemento en el cual fueron contempladas las TD, y específicamente las tecnologías de la información y de la comunicación (TIC), es en el actual Currículo Nacional Básico (CNB), pero estas son abordadas desde una perspectiva tecnicista. Esa presencia se ve manifestada principalmente en el CNB del tercer nivel $\left(7 .^{\circ}, 8 .^{\circ}\right.$ y $\left.9 .^{\circ}\right)$ de educación básica y de los bachilleratos técnicos profesionales (BTP). Es en asignaturas que no corresponden al área de informática, como Sociología e Historia de Honduras, por ejemplo, en las cuales se ve reflejada esa perspectiva tecnicista, colocando a las TIC como simples recursos didácticos para el desarrollo de algunos temas y no como nuevos escenarios de interacción para la producción de conocimiento.

A pesar de existir algunas tentativas por parte del Gobierno de Honduras para la implementación de las TD en el ámbito educativo, este proceso se enfrenta a una serie de factores que condiciona la labor docente. La infraestructura de las escuelas y el acceso a las TD están entre ellos. Esas limitaciones se ven reflejadas en los ambientes escolares, inclusive en los centros educativos de las zonas urbanas, los cuales enfrentan grandes problemas de acceso a las TD e infraestructura inadecuada para el desarrollo de prácticas pedagógicas. Sin embargo, a pesar de las condiciones adversas del espacio físico de los colegios y del limitado acceso a las TD por parte de profesores como de alumnos, en Santa Bárbara se experimenta una variada gama de prácticas pedagógicas con TD, desde las más tecnicistas, como la simple utilización de diapositivas para explicar un tema, hasta prácticas pedagogas innovadoras, como la implementación de simuladores de circuitos electrónicos, entre otras.

5 La Dirección de Tecnologías de la Información y Comunicación (Ditic) es una dependencia de la Comisión Nacional de Telecomunicación (Conatel), la cual cuenta con el portal de transparencia, para el acceso a la información pública mediante el correo electrónico transparencia@conatel.gob.hn. 
Debido a este contexto el presente artículo se enfoca en la discusión de una parte de los resultados de un trabajo de investigación de maestría en educación ${ }^{6}$, cuyo objetivo era comprender los factores que condicionan las prácticas pedagógicas con TD de los profesores de Santa Bárbara, Honduras. Debido a que la infraestructura de los centros educativos y el acceso a TD son dos de los factores que están condicionando la práctica pedagógica, en el presente artículo nos hemos enfocado únicamente en ellos, con el objetivo de comprender de qué manera la infraestructura y el acceso a TD condicionan el desarrollo de prácticas pedagógicas de los profesores de Santa Bárbara.

\section{METODOLOGÍA}

La investigación fue de naturaleza cualitativa, en la cual se procuró tener una posición holística del fenómeno que se estudió. No tomamos un todo para estudiarlo en fragmentos de manera aislada, sino que se estudiaron todos los elementos que hacían parte del fenómeno de estudio y las diferentes interacciones que tienen entre ellos (André, 1995). Esta investigación cualitativa se realizó mediante la implementación de un estudio de caso, porque "cuando se quiere estudiar algo singular, que tenga un valor en sí mismo, debemos escoger el estudio de caso" (Lüdke y André, 1986, p. 17).

La investigación se hizo en cuatro centros gubernamentales de educación media de la ciudad de Santa Bárbara, en el departamento del mismo nombre, en Honduras. El colegio A disponía de tres laboratorios de informática, cada uno de ellos con un aproximado de 20 computadoras portátiles, la matrícula era de 1210 alumnos en el 2016, en 6 modalidades distintas. El colegio $B$ tiene tres laboratorios de informática, cada uno de ellos dispone de un aproximado de 16 computadoras de escritorio, la matrícula para el 2016 fue de 1474 alumnos distribuidos en 9 modalidades. El colegio $C$ cuenta con un solo laboratorio de informática, no informaron la cantidad de computadoras, 788 alumnos en 5 modalidades. El colegio D cuenta con un laboratorio de informática equipado con 50 computadoras, 170 alumnos dispersos en tres modalidades.

En un primer momento se aplicaron cuestionarios a todos los profesores de los cuatro colegios de Santa Bárbara, para seleccionar a los sujetos participantes de la investigación, los cuales debían reunir los siguientes criterios de selección: cantidad de prácticas pedagógicas con TD, frecuencia de implementación de esas prácticas pedagógicas, y que fueran de diferentes áreas académicas. Se seleccionaron ocho profesores que impartían clases en el Bachillerato Técnico Profesional (BTP), a quienes se les realizó una entrevista semiestructurada; en la tabla 1 se presenta una descripción de cada uno

6 Debido a eso el presente texto es una adaptación de una sección del texto original de la investigación, cuyo título es: Labrando Caminos: factores que condicionan las prácticas pedagógicas con tecnologías digitales de los profesores de Santa Bárbara, Honduras. 
de ellos. De igual manera se realizó una observación participante, que abarcó las instalaciones, equipamientos, prácticas pedagógicas, trabajos realizados con los alumnos e interacción en algunos ambientes virtuales.

Tabla 1

Descripción de los ocho profesores participantes de la investigación

\begin{tabular}{|c|c|c|c|c|c|c|c|}
\hline Sujetos & Edad & Sexo & $\begin{array}{c}\text { Años de } \\
\text { experiencia }\end{array}$ & $\begin{array}{l}\text { Área de } \\
\text { trabajo }\end{array}$ & $\begin{array}{c}\text { Área de } \\
\text { formación }\end{array}$ & Modalidad & $\begin{array}{l}\text { Institución de } \\
\text { formación }\end{array}$ \\
\hline \multirow{2}{*}{$\begin{array}{c}\text { Profesor } \\
\text { A1 }\end{array}$} & \multirow{2}{*}{31} & \multirow{2}{*}{ M } & \multirow{2}{*}{10} & \multirow{2}{*}{ Electricidad } & $\begin{array}{c}\text { Técnica } \\
\text { industrial }\end{array}$ & Distancia & UPNFM \\
\hline & & & & & $\begin{array}{l}\text { Ingeniería en } \\
\text { electricidad }\end{array}$ & Presencial & no informado \\
\hline $\begin{array}{c}\text { Profesor } \\
\text { A2 }\end{array}$ & 33 & M & 14 & $\begin{array}{c}\text { Ciencias } \\
\text { naturales }\end{array}$ & $\begin{array}{c}\text { Ciencias } \\
\text { naturales }\end{array}$ & Presencial & UPNFM \\
\hline $\begin{array}{c}\text { Profesor } \\
\text { A3 }\end{array}$ & 38 & M & 16 & Informática & $\begin{array}{c}\text { Matemática e } \\
\text { informática }\end{array}$ & Distancia & UPNFM \\
\hline $\begin{array}{c}\text { Profesor } \\
\text { A4 }\end{array}$ & 35 & M & 12 & $\begin{array}{c}\text { Español y } \\
\text { agropecuaria }\end{array}$ & $\begin{array}{l}\text { Letras } \\
\text { español }\end{array}$ & $\begin{array}{l}\text { Presencial } \\
\text { y Distancia }\end{array}$ & UPNFM \\
\hline $\begin{array}{c}\text { Profesor } \\
\text { B1 }\end{array}$ & 45 & M & 14 & $\begin{array}{l}\text { Mecánica } \\
\text { industrial }\end{array}$ & $\begin{array}{c}\text { Técnica } \\
\text { industrial }\end{array}$ & Distancia & UPNFM \\
\hline $\begin{array}{c}\text { Profesor } \\
\text { B2 }\end{array}$ & 49 & M & 25 & Español & $\begin{array}{c}\text { Gestión de la } \\
\text { educación }\end{array}$ & Distancia & Distancia \\
\hline $\begin{array}{c}\text { Profesor } \\
\text { B3 }\end{array}$ & 40 & $F$ & 18 & Informática & no informado & Distancia & UPNFM \\
\hline $\begin{array}{c}\text { Profesor } \\
\text { B4 }\end{array}$ & 35 & M & $\begin{array}{c}\text { no } \\
\text { informado }\end{array}$ & $\begin{array}{l}\text { Ciencias } \\
\text { contables }\end{array}$ & no informado & Presencial & UNAH \\
\hline
\end{tabular}

Elaboración propia

Aunque se aplicaron los cuestionarios a los cuatro colegios de Santa Bárbara, los demás instrumentos de investigación fueron usados únicamente en dos de ellos. Esto se debió a que los profesores seleccionados en su mayoría trabajaban en los colegios A o B, y alguno de ellos tenía trabajo hasta en dos colegios en diferentes jornadas. Además, los primeros dos colegios cuentan con mejores condiciones de infraestructura en relación con las TD. Los profesores fueron nombrados con la letra referente a la institución donde laboran y un número.

Se procuró buscar un diálogo entre la investigadora y los ocho sujetos participantes de la investigación, para crear la información, independiente de los dispositivos de producción de datos utilizados. Esto permitió identificar elementos que no podrían haber 
sido percibidos mediante la simple implementación de los instrumentos de producción de informaciones. Para lograr este diálogo entre ambas partes fue preciso que la investigadora tuviera una escucha sensible, esta es la forma en la que ella toma conciencia e interfiere en el diálogo con los sujetos sociales, mediante una lógica transversal. La sensibilidad en la escucha es el vínculo de unión entre ambas partes, que se crea de forma elaborada por parte de la investigadora (Barbier, 1998).

Los datos recolectados de las entrevistas fueron analizados a través del análisis textual discursivo, el cual "puede ser comprendido como un proceso autoorganizado de construcción de comprensión en que nuevos entendimientos emergen a partir de una secuencia recursiva de tres componentes" (Moraes y Galiazzi, 2016, p. 34). En dicho proceso se partió de la desconstrucción de los textos, unitarización, para luego establecer las relaciones existentes entre cada uno de los elementos unitarios, que permitió la categorización, para que finalmente pudiera emerger un nuevo metatexto.

Las entrevistas fueron transcritas en su totalidad, para luego ser estudiadas, permitiendo que emergieran las categorías que estaban influyendo en el desarrollo de las prácticas pedagógicas con tecnologías digitales de los profesores de Santa Bárbara. Luego de ser desconstruido el texto de las entrevistas, se pudieron establecer relaciones entre las diferentes categorías resultantes de ellas, las que fueron organizadas por similitudes y, a partir de eso, crear un nuevo metatexto.

\section{RESULTADOS}

Los resultados de la investigación evidenciaron que las actuales condiciones de infraestructura de los colegios de Santa Bárbara, así como el acceso a TD tanto de profesores como de alumnos está dificultando el desarrollo de las prácticas pedagógicas de los profesores. Cada uno de estos factores se ve reflejado de diferentes maneras en el ambiente escolar, influyendo de diferentes maneras.

Infraestructura de los centros educativos. Uno de los aspectos que más sobresale en las discusiones sobre infraestructura en los colegios de Santa Bárbara es el referente a los problemas eléctricos, ya sean estos por viejas instalaciones eléctricas o por los constantes cortes de energía eléctrica en todo el territorio hondureño. A pesar de que la energía eléctrica es uno de los servicios públicos más distribuidos en Latinoamérica, aún existen comunidades que no tienen acceso a este servicio, principalmente en Centro América y Perú (Duarte, Gargiulo y Moreno, 2011). En el caso de los cuatro colegios de Santa Bárbara, sí cuentan con acceso a energía eléctrica, pero presentan problemas en la calidad del servicio, debido a los constantes apagones o cortes del fluido eléctrico, lo que termina desencadenando el deterioro de otros recursos materiales de los centros educativos. 
A raíz del problema de acceso a energía eléctrica se han dañado algunos dispositivos electrónicos de los centros educativos, lo que viene a empeorar su precaria situación, pues se dispone de pocos recursos materiales para el desarrollo de las clases. Ante el miedo de continuar dañando otros dispositivos electrónicos, como las pizarras digitales, en el colegio B han dejado de utilizarlas. La profesora B3 manifestaba que para poder usar ese recurso era necesario desconectar los aparatos eléctricos dentro del laboratorio de informática para que así pudiera funcionar, debido al bajo voltaje de energía, por lo que eso solo lo hicieron durante el primer año, posteriormente suspendieron su empleo para evitar que se averiase por un apagón. Esta situación se sale completamente del control de las autoridades educativas del colegio o de la Secretaría de Educación, ya que dependen de la calidad del servicio que ofrece la única empresa de energía eléctrica en el territorio, que a partir del año 2015 fue semiprivatizada, por lo que tanto la Empresa de Energía Eléctrica (ENEE), que pertenece al Estado, como la Empresa de Energía Honduras (EEH), de capital privado, deben buscar soluciones que garanticen la calidad del servicio para toda la población hondureña.

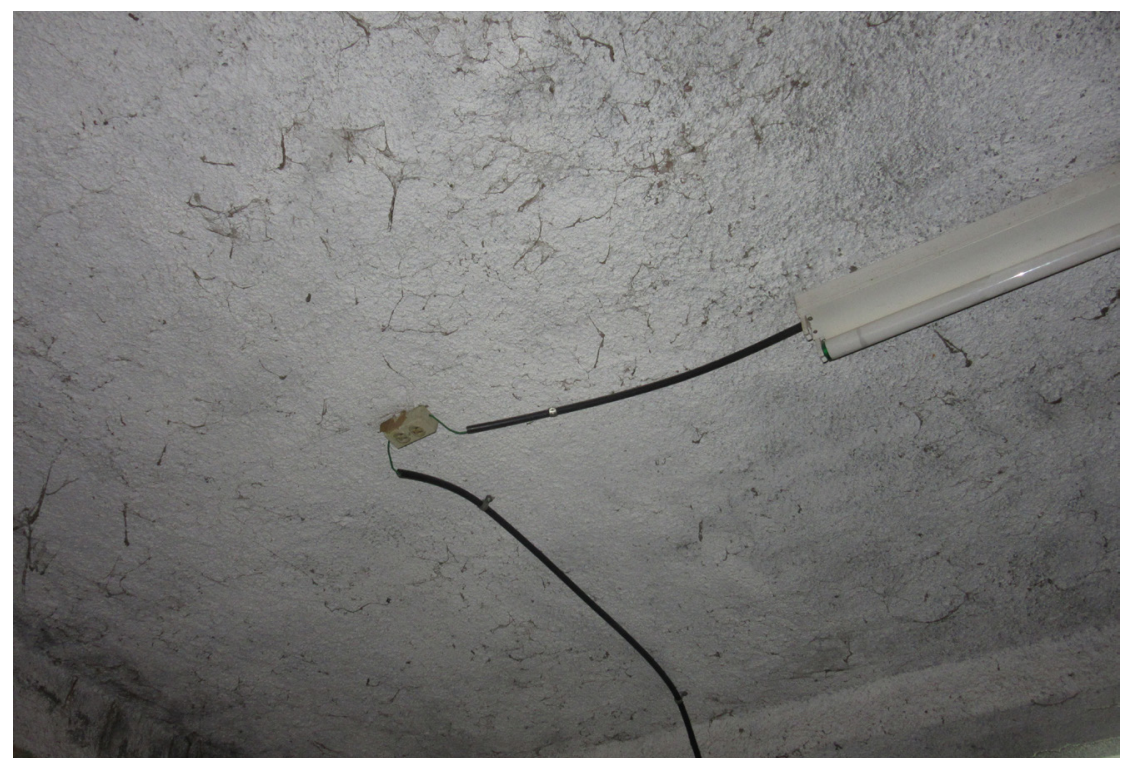

Figura 1. Toma de energía eléctrica en el colegio $B$

Fotografía: Archivo de las autoras

Además de los problemas con el fluido eléctrico, los colegios no cuentan con instalaciones eléctricas adecuadas para el desarrollo de prácticas pedagógicas con TD. En el caso del colegio $B$, las tomas eléctricas prácticamente son inexistentes en las aulas 
de clases. Pocas son las que sí disponen de ellas, pero estas se encuentran ubicadas en los techos, lo que dificulta, evidentemente, que los profesores puedan conectar sus dispositivos digitales, sean estos proyectores, equipos de sonido o simplemente cargar un teléfono o sus computadoras portátiles. En la figura 1 se puede apreciar una de las tomas eléctricas en el techo de un aula de clases del colegio B. Algo similar sucede en el colegio $A$, en donde no todas las aulas tienen acceso a energía eléctrica. En el caso del profesor A4, se ve en la necesidad de llevar extensiones eléctricas para transportar la energía eléctrica del aula de clases más próxima a la suya. Lógicamente, esto lo realiza solo cuando el desarrollo de algunas de sus clases depende en gran medida de la implementación de algún dispositivo eléctrico. Este tipo de instalaciones improvisadas no están permanentemente, lo que dificulta que cualquier persona pueda tener acceso a este servicio en ese espacio del colegio. Cabe destacar que el aula de clases se encuentra en reformas, las cuales son realizadas por iniciativa del profesor, pero no se pudo observar que se estén incluyendo las tomas eléctricas para que en el futuro se pueda contar con este servicio.

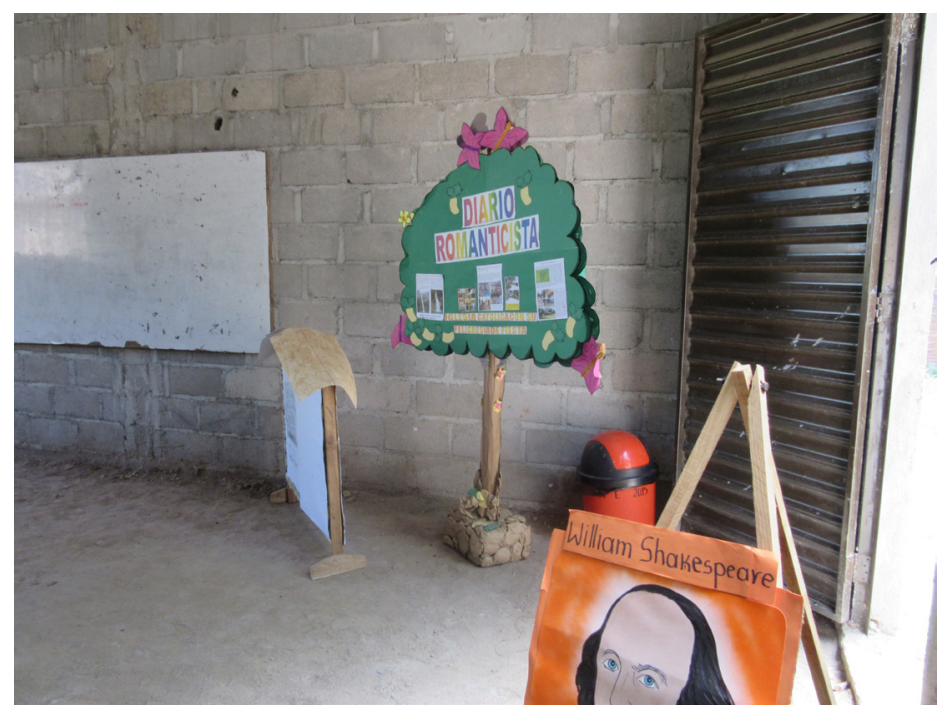

Figura 2. Aula de clases en el colegio A

Fotografía: Archivo de las autoras

Otro aspecto importante referente a los edificios de los centros educativos es que son viejos. Específicamente en el colegio $\mathrm{A}$, se le ha dado mantenimiento a lo largo de los años, sin embargo, como se construyó en la década de los 70, en la cual la presencia de tantos dispositivos eléctricos no era tan común, no cuenta con el diseño apropiado para la realidad actual. Esto se evidencia notablemente en los laboratorios de informática, 
que inicialmente eran aulas de clases. Fueron diseñadas con esa intención, pero ante la necesidad de tener un espacio físico para un laboratorio de este tipo, fue necesario reacondicionarlas. En lo referente a la llegada de las TD a los ambientes escolares, no solo tomó de sorpresa a los profesores sobre cómo implementarlas en las prácticas pedagógicas, sino que también se evidenció que no estaban preparados en infraestructura para esa eminente presencia y protagonismo.

Todos los espacios físicos de las instituciones educativas deben ser reconfigurados, diseñados en función de las demandas de la sociedad contemporánea, en donde existe un gran protagonismo de las TD. Podemos observar cómo otros espacios físicos en la sociedad han realizado esa readecuación. Por ejemplo, los centros comerciales, aeropuertos, restaurantes, cafés, cuentan con instalaciones para que las personas que frecuentan estos espacios puedan cargar sus diversos dispositivos digitales, pero ese reacondicionamiento no está sucediendo de igual manera en los espacios educativos, en los cuales cargar un celular, o inclusive una computadora, resulta difícil, lo que complica la conexión en red en los ambientes digitales por parte de los actores escolares.

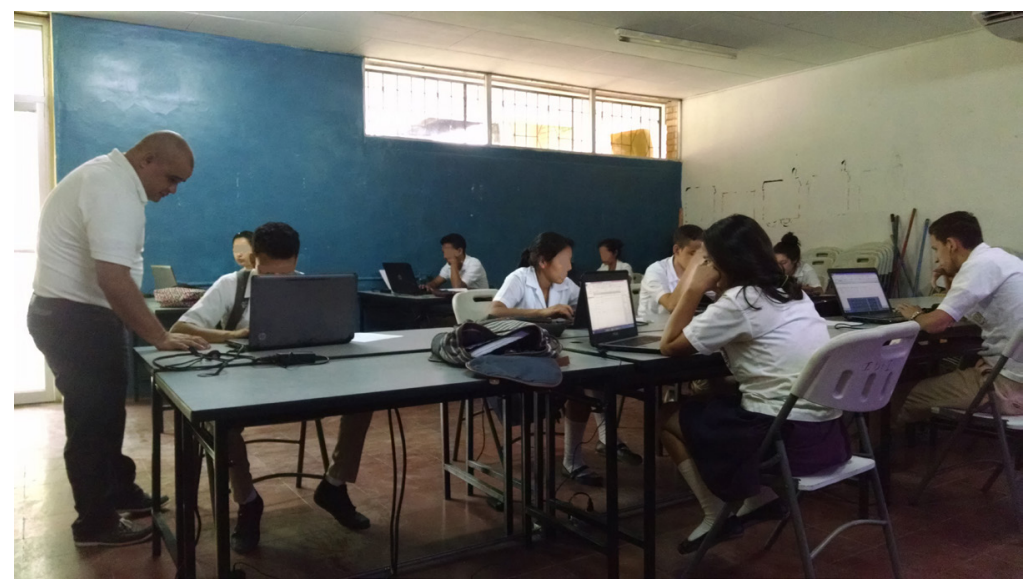

Figura 3. Laboratorio de informática del colegio A

Fotografía: Archivo de las autoras

Equipamiento de tecnologías digitales. El equipamiento de TD en los colegios de Santa Bárbara presenta grandes dificultades para el mantenimiento/actualización, e inclusive marcadas diferencias entre las instituciones, como en el caso del colegio A, que dispone de equipos más modernos, como se puede apreciar en la figura 3. Los alumnos utilizan computadoras portátiles en los laboratorios de informática, pero esto no sucede en los demás colegios. En el caso del colegio B, los laboratorios de informática fueron creados con el Programa de Apoyo a la Enseñanza Media en Honduras (Praemoh), por parte de 
la Secretaría de Educación de Honduras, sin embargo, las computadoras con las cuales fueron equipados estos espacios se han ido deteriorando con el paso de los años, como se puede aprecia en la figura 4, los monitores son obsoletos, los CPU han tenido que ser reparados con piezas de otros CPU. Las autoridades educativas, desarrollan proyectos encaminados a la incorporación de las TD en los ambientes educativos, pero estos no tienen una continuidad y queda bajo la iniciativa de profesores, alumnos y padres de familia el dar mantenimiento. Al igual que sucede en Brasil, en Honduras se implementan ciertos programas aislados, que se desvinculan de otras políticas públicas, pero sobre todo alejadas de las realidades que experimentan las sociedades (Bonilla y Pretto, 2011).

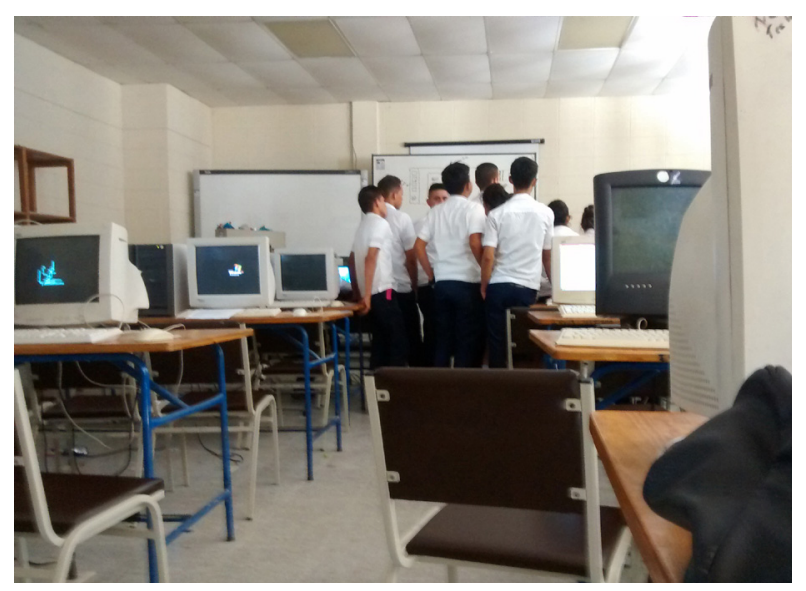

Figura 4. Laboratorio de informática del colegio B

Fotografía: Archivo de las autoras

El colegio B, además de tener equipos obsoletos, no dispone de otras tecnologías digitales importantes (como proyectores, por ejemplo), para el desarrollo de las actividades académicas. En tal sentido, si asignaturas directamente relacionadas con lo tecnológico-digital presentan problemas de acceso en materia de equipamiento (tal cual ocurre con informática), el panorama se torna más desalentador en áreas como español, estudios sociales, etc., en las cuales suele predominar una percepción según la cual el uso/acceso a las TD se valora de manera accesoria o prescindible, lo cual desestimula la presencia de los recursos digitales en tales disciplinas. Por ejemplo, el profesor A1 intentó implementar software de simulación de circuitos eléctricos en sus prácticas pedagógicas, pero estas se han visto dificultadas debido a que el taller de electricidad no dispone de computadoras, por lo que el profesor se ve en la necesidad de emplear su propia laptop para que los alumnos puedan practicar con el software y, posteriormente, poner en práctica el conocimiento aprendido en situaciones reales. 
La condición de infraestructura de los colegios de Santa Bárbara dificulta que las TD sean implementadas en las prácticas pedagógicas, lo que genera una exclusión digital de los alumnos, para los cuales la escuela puede representar un espacio importante para interaccionar en los ambientes digitales. Estas mismas condiciones de infraestructura escolar determina en gran medida el acceso de los profesores y alumnos a las TD dentro de los colegios.

Acceso a tecnologías digitales. La exclusión social que permea en Honduras la podemos ver plasmada en el contraste entre los colegios $A$ y $B$, los cuales tienen condiciones de infraestructura diferentes, dependiendo, en gran medida, de las características socioeconómicas de los alumnos de cada centro educativo. Muchos de los centros educativos disponen de poca o casi nula ayuda por parte de la Secretaría de Educación de Honduras, por lo que el mantenimiento de la infraestructura, incluyendo la adquisición de nuevos recursos didácticos (como los recursos digitales) depende en gran medida de iniciativas de las autoridades institucionales, profesores, alumnos y padres de familia. En el caso del colegio A, que es de jornada matutina, asisten alumnos con mejores condiciones económicas, lo que posibilita que la institución reciba el apoyo de padres/ madres de familia en actividades con fines lucrativos, sin embargo, en el colegio B, cuyas jornadas son vespertina/nocturna, asisten alumnos con un perfil socioeconómico diferente, muchos de ellos trabajan media o jornadas completas para costear sus estudios y, evidentemente, el apoyo hacia la institución no es tan fuerte como en el anterior.

La exclusión social genera dificultades para tener acceso a TD, tanto por parte de los alumnos como de los profesores de Santa Bárbara. A pesar de que los profesores están conscientes de la importancia que tienen las TD para el desarrollo de sus prácticas pedagógicas, se les dificulta el implementarlas debido al poco acceso que tienen a ellas. No existe una política pública en Honduras que permita que los profesores tengan acceso a esas TD, y a esto se le suma la dificultad que significa el adquirir una TD con recursos propios debido a sus altos costos y los bajos salarios. El profesor A4 expresó: “[...] pero es bien difícil al final, porque uno dice: pucha son 14000 , es tanto de dinero que voy a invertir, y para unas pocas clases [...]" (Profesor A4, comunicación personal, 16 de junio del 2016), al referirse a la posibilidad de comprar un proyector para implementarlo en su desempeño docente.

El acceso a TD es un derecho que solo aquellos con cierto nivel de ingresos económicos puede tener. En la actualidad el mercado de las TD no incluye a los grupos sociales pobres que tienen bajos ingresos económicos. Eso lo podemos ver reflejado en el caso de Honduras en donde el alto costo de las TD dificulta que un gran sector de la sociedad, incluidos los profesores, pueda tener acceso a ellas. En el caso de los profesores de educación media, la hora clase tiene un valor de L66,58 (equivalente a \$2,70 aproximadamente), y un profesor de educación primaria sin título universitario devenga L10 
$863,88(\$ 441,98)$ mensualmente. Si contrastamos ese salario con los precios de algunas TD, disponibles en la tabla 2, podemos verificar que, para tener acceso a internet en casa, un profesor de primaria tendría que destinar el 9,95\% de su salario. Esto evidencia que los problemas de acceso a TD en Honduras están ligados directamente a problemas sociales, de exclusión social, y mientras no se piensen en conjunto ambos problemas, difícilmente podremos disminuir las brechas existentes.

Tabla 2

Precios de algunas TD en Honduras en el 2019

\begin{tabular}{lcc}
\hline \multicolumn{1}{c}{ Tecnologías digitales } & Valor mínimo & Valor máximo \\
\hline Planes de internet en el celular & $\$ 19,99$ de $3,5 \mathrm{~GB}$ & $\$ 89,99$ de $25 \mathrm{~GB}$ \\
Planes de internet en casa & $\$ 44$ de $10 \mathrm{MB}$ & $\$ 55$ de $20 \mathrm{MB}$ \\
Computador & $\$ 300$ & $\$ 2800$ \\
Celular & $\$ 80$ & $\$ 7720$ \\
Proyector & $\$ 200$ & $\$ 720$ \\
\hline
\end{tabular}

Elaboración propia

El poco o inclusive nulo acceso a TD representa también una dificultad para acceder a las informaciones disponibles en los ambientes digitales. "Quien está desconectado desconoce el océano informacional, quedando imposibilitado de encontrar una información básica, de descubrir nuevos temas, de despertar para nuevos intereses" (Silveira, 2001, p. 17). Al ser difícil tener esas informaciones, también se dificulta el atribuirles un significado, no se permite la creación de conocimientos y se impide que los individuos puedan difundirlos. En la sociedad contemporánea, el conocimiento y la información han adquirido grandes valores, inclusive comerciales; el dificultar o imposibilitar que algunos grupos sociales los tengan representa una exclusión social. La segregación social que se da en la distribución de la riqueza también se ve plasmada en la distribución y acceso a las TD. Las personas de los grupos más adinerados tienen acceso a todos los recursos digitales, desde la comodidad de sus casas. Cuentan con computadores, celulares, internet, etc., lo que les posibilita el tener acceso a las informaciones, poder producir y compartirlas en la red. En el otro extremo están los grupos más empobrecidos, que no logran tener acceso a esas tecnologías en casa, quedando aislados de las informaciones y de las producciones compartidas en la red (Bonilla y Pretto, 2011; Silveira, 2001). 
Sin embargo, a pesar de los salarios bajos y el alto costo de las TD en Honduras, los profesores han conseguido algunas de ellas con recursos propios, ante la inexistencia de una política pública que los apoye. De los profesores que respondieron el cuestionario, el $98,18 \%$ dispone de internet en casa, un $84,34 \%$ tiene una laptop en su hogar y $83,13 \%$ de los profesores posee teléfonos inteligentes, por lo que se puede inferir que una gran mayoría de ellos tiene acceso a TD. Esto constituye un factor que posibilita la implementación de las TD en las prácticas pedagógicas.

En el caso de los estudiantes en los colegios de Santa Bárbara, el panorama es muy diferente. Para la mayoría es difícil tener acceso a las TD. Según el informe "Estado de la banda ancha en América Latina y el Caribe 2017", Honduras se encuentra entre los países con menos acceso a internet en casa, con un porcentaje de $25 \%$ de hogares que disponen de este servicio, en contraste del $7 \%$ aproximadamente del 2010 (Cepal, 2018). Aunque se ha venido avanzando en este tema, aún no es suficiente; en el 2014 se implementó la Agenda Digital Honduras 2014-2018, dirigida a la promoción de la competitividad e innovación a través del uso efectivo, masivo y de calidad de las TIC, con la intención de reducir la brecha digital existente en el país, buscando la eficiencia del gobierno, la cercanía con la población para lograr una economía más competitiva y desarrollada. Se enfoca en cuatro ejes estratégicos: conectividad digital con equidad, gobierno digital, talento humano en TIC y desarrollo del marco institucional y regulatorio.

Ante el hecho de que no se han podido superar las limitaciones de acceso a TD en el país, los estudiantes de Santa Bárbara buscan alternativas para acceder a ellas. Una de esas alternativas son los ciber, en los cuales los estudiantes pagan para tener acceso a internet, computadora e incluso videojuegos. El promedio del valor de una hora de internet en Santa Bárbara es de L.15 (equivalente a \$0,21), que no todos pueden pagar. Estos espacios, de naturaleza privada, permiten que los jóvenes puedan tener acceso a internet e interaccionar en los ambientes digitales, para la realización de las tareas que son asignadas por los profesores, lo que permite que los docentes puedan incorporar las tecnologías digitales en sus prácticas pedagógicas.

Ante este panorama, las escuelas podrían representar un espacio en el cual se facilite que tanto alumnos como profesores tengan acceso a las TD. En el caso de Santa Bárbara, los cuatro centros de educación media públicos tienen acceso a algunas TD, sobre todo a internet, aunque este servicio, que está siendo proporcionado por parte del gobierno mediante el proyecto El Internet del Pueblo, presenta algunos inconvenientes, como la libertad de acceso a determinados sitios en internet y el ancho de banda, que no da cuenta de la cantidad de alumnos en las instituciones educativas. Al ser este servicio centralizado con altos estándares de seguridad y filtrado de contenido, se impide el acceso a diversos sitios web. Básicamente, los profesores lo usan para ingresar las calificaciones al Sistema de Administración de Centros Educativos (SACE). Estos mecanismos 
de control dificultan el libre acceso a la información y al proceso de democratización del conocimiento, pues es necesario que no existan filtros para fomentar el trabajo colaborativo en los colegios.

Además de las evidentes limitaciones del proyecto El Internet del Pueblo, referentes a la restricción de acceso a determinados sitios en internet y del ancho de banda, también se enfrenta a las políticas institucionales que obstaculizan el acceso a este servicio, debido a que no permiten que sea abierto para todos los alumnos. Esto demuestra una desvinculación de las políticas públicas con las políticas institucionales. Una busca disminuir la brecha digital y la otra bloquea que los alumnos tengan un acceso libre al internet en los centros educativos, por lo que, finalmente, las instituciones gubernamentales terminan presentando en sus informes resultados de acceso a internet que no se ven reflejados en la realidad. Por ejemplo, en el colegio B existía un servicio de internet proporcionado de manera gratuita por una institución, pero fue retirado por mandato de las autoridades escolares, porque estas consideraban que los profesores se distraían mucho, lo que impedía que se enfocaran en sus funciones docentes.

Estas medidas restringen el acceso a TD y obstaculizan su implementación en las prácticas pedagógicas. Esto se debe a que las políticas públicas se enfocan en el equipamiento de los laboratorios de informática en los centros educativos, pero dejan de lado lo referente a la formación de los actores escolares para la interrelación en un ambiente de la cultura digital dentro de las escuelas (Silva, 2014). Es fundamental formar tanto al personal docente como al administrativo, para que en conjunto puedan establecer políticas institucionales que permitan la libre implementación de TD en las prácticas pedagógicas.

Siendo que el servicio de El Internet del Pueblo no genera las mejores condiciones para el acceso a información, los centros educativos se ven en la necesidad de contratar los servicios de internet de empresas privadas, lo cual es financiado por los alumnos. Pero ambos servicios son de uso exclusivo para las clases de informática, y otros profesores, de áreas académicas diferentes, no acceden a estos laboratorios, y por ende al internet.

Los laboratorios de informática están destinados para las clases de informática o disciplinas afines, y no para que profesores de otras áreas académicas puedan hacer uso de ese espacio para el desarrollo de determinada clase. Esto se vio reflejado en el discurso de muchos profesores, como el profesor B2 que manifestó: "[...] limitan a que deben usarse solamente a nivel del laboratorio, no que el alumno lo pueda tener en sus teléfonos, o sea, no hay que dar la clave, porque solamente es para que se use en el laboratorio [...]" (Profesor B2, comunicación personal, 7 de junio del 2016). Sin la libertad de tener acceso a informaciones, e incluso de hacerlas disponibles, resulta difícil que los alumnos y profesores puedan vivenciar una cultura digital dentro de sus centros educativos. 
Es una lucha por lograr la democratización del conocimiento, una construcción colectiva de las informaciones y de conocimientos que se ve amenazada por los códigos y normas cerradas. Para lograr tal democratización, es fundamental pensar en políticas públicas e institucionales que garanticen no solo el acceso a los dispositivos digitales, también la conexión a internet, y no cualquier conexión, sino una de alta velocidad que permita tener un mayor acceso/difusión de las producciones multimedia, que van en aumento en los últimos años debido al auge de los dispositivos digitales móviles (Pretto y Silveira, 2008).

A pesar de estas limitaciones de acceso a TD dentro y fuera de los centros educativos, los profesores las implementan en sus prácticas pedagógicas dentro de sus posibilidades como las de sus alumnos. Evidentemente, las limitaciones antes mencionadas no permiten que se aprovechen todas las potencialidades de las TD, ni vivenciar la cultura digital en los ambientes escolares. Pero, las mismas características de las TD permiten que se puedan vencer en cierta manera las limitaciones del espacio y tiempo, por lo que de una manera u otra terminan inscribiéndose en la práctica pedagógica.

\section{CONCLUSIONES}

Las prácticas pedagógicas son una práctica social que se realiza con la intencionalidad de lograr un proceso pedagógico, que debe caminar hacia la obtención de una transformación colectiva, convirtiéndola en una práctica educativa, que integre diferentes elementos sociales para alcanzar un proceso educativo. Esto se hace cada vez más necesario ante la presencia y beligerancia de las tecnologías digitales en la cotidianidad social de los seres humanos, las cuales también se hacen presentes en los ambientes educativos, a través de diferentes medios, permitiendo que emerjan diversas prácticas pedagógicas con TD.

La implementación de las TD en las prácticas pedagógicas de los profesores de Santa Bárbara se ha visto condicionada por una serie de factores, interfiriendo o facilitándolas de múltiples formas. Factores que van desde un contexto macro de Honduras, como los problemas de acceso a energía eléctrica, pasando por contextos locales e institucionales, como las políticas públicas e institucionales, hasta llegar a los de índole individual, como la percepción de los profesores sobre las TD. Todo este panorama condicionante hace que los profesores de Santa Bárbara realicen prácticas pedagógicas desde una perspectiva tecnicista y otros desde una de innovación de la práctica pedagógica aprovechando las potencialidades de las TD.

Los problemas macros, como los del acceso a energía eléctrica, no solo están afectando al sistema educativo en el país, sino que todos los sectores se ven perjudicados, ante un servicio inestable. Estas limitaciones para el desarrollo de prácticas pedagógicas con TD deben ser abordadas desde una política pública, que permita el tener acceso a 
este servicio de una manera más estable y eficiente, buscando otros mecanismos de generación de energía eléctrica que puedan satisfacer la demanda del país.

Siempre en el contexto macro, vemos cómo la exclusión digital se debe abordar también como una exclusión social, ya que los alumnos con bajos recursos económicos no tienen acceso a todos los recursos tecnológicos en sus escuelas o en sus hogares, lo que dificulta que los profesores puedan implementar las TD en sus prácticas pedagógicas. Es necesario que tanto alumnos como profesores tengan acceso a TD fuera de las instalaciones escolares, para que puedan interaccionar en la cultura digital en todos los ambientes físicos en los que se desplazan. Mientras no se logre vencer las brechas sociales en Honduras, difícilmente se podrá caminar hacia una verdadera inclusión digital del pueblo hondureño.

El poco acceso a TD por parte de los alumnos se ve condicionado principalmente por los altos costos. Inclusive para los profesores es difícil adquirir con dinero propio muchos recursos tecnológicos. Esto, junto a los bajos salarios de los profesores, impide que se experimenten prácticas pedagógicas con TD, por lo que solo se implementan aquellas a las que pueden tener acceso por iniciativa propia. La falta de una política pública para facilitar que los profesores de Honduras tengan acceso a tecnologías digitales a un bajo costo representa un factor en contra de la incorporación de las tecnologías digitales en las prácticas pedagógicas.

Aunque se han venido implementando algunas políticas públicas encaminadas a la inclusión digital, como el proyecto El Internet del Pueblo, estas no están contribuyendo de una manera significativa a que se pueda vivenciar la cultura digital, tanto fuera como dentro de los centros educativos, y esto se debe principalmente a que se está ofreciendo un servicio con muchas restricciones de acceso, pero sobre todo de un ancho de banda insuficiente. Otro ejemplo de los problemas de las iniciativas gubernamentales son los equipamientos de computadoras en los centros educativos, mediante programas como el de Praemho (Programa de Apoyo a la Enseñanza Media en Honduras), pues estas no les dan continuidad ni acompañamiento a los proyectos, por lo que, con el tiempo, los equipos se van quedando obsoletos, y no es posible que TD modernas y actualizadas se vayan implementando en las prácticas pedagógicas.

Al no darse una continuidad o acompañamiento por parte de las autoridades educativas, las condiciones de infraestructura en los colegios de Santa Bárbara impiden que se den prácticas pedagógicas con TD. El no disponer de las condiciones adecuadas para utilizar las tecnologías digitales dentro de los salones de clase evita que se dé un proceso de innovación en las prácticas educativas. Además del problema de infraestructura, el equipo que los colegios tienen muchas veces está en mal estado, y no es suficiente para la población estudiantil, por lo que los laboratorios de informática son destinados exclusivamente para las clases afines al área de Informática. 
La implementación de las TD en las prácticas pedagógicas se ve perjudicada por la poca articulación de las políticas públicas e institucionales, las cuales en ocasiones caminan en direcciones opuestas. Las pocas iniciativas por parte de las autoridades educativas en Honduras se ven limitadas por políticas institucionales que hacen que el acceso a las pocas TD disponibles en los centros educativos no esté abierto para todo el personal docente y alumnado.

Para formar a los individuos con las habilidades que demanda la sociedad contemporánea es necesario que los centros educativos en Honduras permitan vivenciar activamente la cultura digital, mediante políticas públicas e institucionales de libre acceso a TD, formación de profesores, que permitan que las prácticas pedagógicas con TD sean parte de la praxis, es decir dentro de una práctica social. Aún queda mucho camino por recorrer y labrar en los colegios de Santa Bárbara, Honduras, pero el recorrido ya se ha iniciado, y, con proyectos de formación y creación de políticas de acceso a tecnologías digitales, ese camino puede tomar destinos más optimistas.

\section{REFERENCIAS}

André, M. E. D. A. De. (1995). Etnografía da Prática Escolar. Campinas, SP: Papirus.

Barbier, R. (1998). Escuta Sensivél na Abordagem Transversal. En BARBOSA, Joaquim Golçalves (coord.). Multirreferencialidade nas Ciências e na Educação. São Carlos: EdUFSCar.

Barragán, D. F. (2012). La práctica pedagógica: pensar más allá de las técnicas. En Barragán, D.F., Gamboa, A. A. y Urbina, J. E. Práctica Pedagógica: Perspectivas Teóricas (1. a ed.). Bogotá: Eco Ediciones.

Bonilla, M. H. S. y Pretto, N. L. (2011). Inclusão digital: polêmica contemporânea (Vol. 2). Salvador: EDUFBA.

Comisión Económica para América Latina y el Caribe (Cepal). (2018). Estado de la Banda Ancha en América Latina y el Caribe. Santiago de Chile: Naciones Unidas.

Duarte, J., Gargiulo, C. y Moreno, M. (2011). Infraestructura escolar y aprendizajes en la educación básica latinoamericana: Un análisis a partir del SERCE. Washington, D.C.: Banco Interamericano de Desarrollo.

Franco, M. A. do R. S. (2016). Práctica pedagógica e docência: um olhar a partir da epistemologia do conceito. Revista Brasileira de Estudos Pedagógicos, 97(247), p. 534-551. Recuperado de http://rbep.inep.gov.br/index.php/rbep/article/ view/2882/pdf 
Lüdke, M. y André, M. E. (1986). A pesquisa em Educação: abordagens qualitativas. São Paulo: EPU.

Moraes, R. y Galiazzi, M. do C. (2016). Análise Textual Discursiva (3. ed). ljuí: Ed. Unijuí.

Organización de las Naciones Unidas para la Educación, la Ciencia y la Cultura, Unesco. (2006). Segundo Estudio Regional Comparativo y Explicativo (SERCE). Recuperado de https://unesdoc.unesco.org/ark:/48223/pf0000160659

Pretto, N. y Silveira, S. A. da. (2008). Além das redes de colaboração: internet, diversidade cultural e tecnologias do poder. Salvador EDUFBA.

República de Honduras. (2010). República de Honduras Visión de País 2010-2038 y Plan de Nación 2010- 2022. Tegucigalpa: [s.n.]. Recuperado de https://eeas.europa.eu/ sites/eeas/files/lc_10.pdf

SACE. (s. f.). Sistema de Administración de Centros Educativos. [página web]. Recuperado de https://sace.se.gob.hn

Silva, M. L. G. da. (2014). A inclusão digital nas políticas públicas de inserção das Tecnologias de Informação e Comunicação na Educação: o discurso e a prática dos cursos de formação de professores. (Tesis de maestría). Faculdade de Educação da Universidade Federal da Bahia. Salvador.

Silveira, S. A. da. (2001). Exclusão Digital: a miséria na era da informção (1. ed.). São Paulo: Fundação Perseu Abramo. 